\title{
NODAL SOLUTIONS FOR THE FRACTIONAL YAMABE PROBLEM ON HEISENBERG GROUPS
}

\author{
ALEXANDRU KRISTÁLY \\ Dedicated to Professor Patrizia Pucci on the occasion of her 65th birthday
}

\begin{abstract}
We prove that the fractional Yamabe equation $\mathcal{L}_{\gamma} u=|u|^{\frac{4 \gamma}{Q-2 \gamma}} u$ on the Heisenberg group $\mathbb{H}^{n}$ has $\left[\frac{n+1}{2}\right]$ sequences of nodal (sign-changing) weak solutions whose elements have mutually different nodal properties, where $\mathcal{L}_{\gamma}$ denotes the CR fractional sub-Laplacian operator on $\mathbb{H}^{n}, Q=2 n+2$ is the homogeneous dimension of $\mathbb{H}^{n}$, and $\gamma \in \bigcup_{k=1}^{n}\left[k, \frac{k Q}{Q-1}\right)$. Our argument is variational, based on a Ding-type conformal pullingback transformation of the original problem into a problem on the CR sphere $S^{2 n+1}$ combined with a suitable Hebey-Vaugon-type compactness result and group-theoretical constructions for special subgroups of the unitary group $\mathbf{U}(n+1)$.
\end{abstract}

\section{INTRODUCTION}

After the seminal paper of Caffarelli and Silvestre [9], considerable efforts have been made concerning the study of elliptic problems involving the fractional Laplace operator both in Euclidean and non-Euclidean settings. As expected, the Euclidean framework is much more developed; although many results concerning the pure Laplace operator can be transposed to the fractional setting in $\mathbb{R}^{n}$, there are also subtle differences which require a deep understanding of certain nonlinear phenomena, see e.g. Cabré and Sire [6], Caffarelli [7], Caffarelli, Salsa and Silvestre [8], Di Nezza, Palatucci and Valdinoci [10], and references therein.

By exploring analytical and spectral theoretical arguments, important contributions have been obtained recently within the CR setting concerning the fractional Laplace operator with various applications in sub-elliptic PDEs, see Branson, Fontana and Morpurgo [5], Frank and Lieb [14], and Frank, del Mar González, Monticelli and Tan [15]. In particular, in the latter papers sharp Sobolev and Moser-Trudinger inequalities are established on the Heisenberg group $\mathbb{H}^{n}, n \geq 1$, the simplest non-trivial CR structure.

In the present paper we shall consider the fractional Yamabe problem on the Heisenberg group $\mathbb{H}^{n}$, namely,

$$
\left\{\begin{array}{l}
\mathcal{L}_{\gamma} u=|u|^{\frac{4 \gamma}{Q-2 \gamma}} u \text { on } \mathbb{H}^{n}, \\
u \in D^{\gamma}\left(\mathbb{H}^{n}\right) .
\end{array}\right.
$$

$(\mathbf{F Y H})_{\gamma}$

Hereafter, $Q:=Q_{n}=2 n+2$ is the homogeneous dimension of $\mathbb{H}^{n}, \gamma>0$ is a parameter specified later, $\mathcal{L}_{\gamma}$ denotes the $\mathrm{CR}$ fractional sub-Laplacian operator on $\mathbb{H}^{n}$, and the functional space $D^{\gamma}\left(\mathbb{H}^{n}\right)$ contains real-valued functions from $L^{\frac{2 Q}{Q-2 \gamma}}\left(\mathbb{H}^{n}\right)$ whose energy associated to the CR fractional sub-Laplacian operator $\mathcal{L}_{\gamma}$ is finite; see $\S 2.4$ for details.

Due to the recent paper of Frank, del Mar González, Monticelli and Tan [15], we know the existence of positive solutions of $(\mathbf{F Y H})_{\gamma}$ for $\gamma \in(0, Q / 2)$, having the form

$$
u(z, t)=c_{0}\left(\left(1+|z|^{2}\right)^{2}+t^{2}\right)^{\frac{2 \gamma-Q}{4}}, \quad(z, t) \in \mathbb{H}^{n},
$$

for some $c_{0}>0$, allowing any left translation and dilation. In the special case $\gamma=1$, when $\mathcal{L}_{1}=\mathcal{L}$ is the usual sub-Laplacian operator on $\mathbb{H}^{n}$, the existence and uniqueness (up to

2000 Mathematics Subject Classification. Primary 35R03; Secondary 35B38.

Key words and phrases. CR fractional sub-Laplacian; nodal solution; Heisenberg group. 
left translation and dilation) of positive solutions of the form (1.1) for problem $(\mathbf{F Y H})_{1}$ have been established by Jerison and Lee [18, 19]; see also Garofalo and Vassilev [16] for generic Heisenberg-type groups (e.g. Iwasawa groups).

Our main result guarantees sign-changing solutions for the fractional Yamabe problem $(\mathbf{F Y H})_{\gamma}$ as follows:

Theorem 1.1. Let $\gamma \in \bigcup_{k=1}^{n}\left[k, \frac{k Q}{Q-1}\right)$, where $Q=2 n+2$. Then problem $(\mathbf{F Y H})_{\gamma}$ admits at least $\left[\frac{n+1}{2}\right]$ sequences of sign-changing weak solutions whose elements have mutually different nodal properties. (Hereafter, $[r]$ denotes the integer part of $r \geq 0$.)

Before commenting on Theorem 1.1 we recall that similar results are well known in the Euclidean setting; indeed, Bartsch, Schneider and Weth [3] proved the existence of infinitely many sign-changing weak solutions for the polyharmonic problem

$$
\left\{\begin{array}{l}
(-\Delta)^{m} u=|u|^{\frac{4 m}{N-2 m}} u \text { in } \mathbb{R}^{N} \\
u \in \mathcal{D}^{m, 2}\left(\mathbb{R}^{N}\right)
\end{array}\right.
$$

where $N>2 m, m \in \mathbb{N}$, and $\mathcal{D}^{m, 2}\left(\mathbb{R}^{N}\right)$ denotes the usual higher order Sobolev space over $\mathbb{R}^{N}$. In fact, their proof is based on Ding's original idea, see [11], who considered the case $m=1$, by pulling back the variational problem $(\mathbf{P})_{m}$ to the standard sphere $S^{N}$ by stereographic projection. In this manner, by exploring certain properties of suitable subgroups of the orthogonal group $\mathbf{O}(N+1)$, the authors are able to obtain compactness by exploring a suitable Sobolev embedding result of Hebey and Vaugon [17] which is indispensable in the application of the symmetric mountain pass theorem.

We notice that sign-changing solutions are already guaranteed to the usual CR-Yamabe problem $(\mathbf{F Y H})_{1}$ by Maalaoui and Martino [20], and Maalaoui, Martino and Tralli [21] by exploring Ding's approach; their results are direct consequences of Theorem 1.1 for $\gamma=1$.

Coming back to Theorem 1.1, we shall mimic Ding's original idea as well, emphasizing that our $C R$ fractional setting requires a more delicate analysis than either the polyharmonic setting in the Euclidean case (see [3]) or the usual CR framework, i.e., when $\gamma=1$ (see $[20,21]$ ). In the sequel we sketch our strategy. As expected, we first consider the fractional Yamabe problem on the CR sphere $S^{2 n+1}$, i.e.,

$$
\left\{\begin{array}{l}
\mathcal{A}_{\gamma} U=|U|^{\frac{4 \gamma}{Q-2 \gamma}} U \text { on } S^{2 n+1}, \\
U \in H^{\gamma}\left(S^{2 n+1}\right),
\end{array}\right.
$$

where the intertwining operator $\mathcal{A}_{\gamma}$ and Sobolev space $H^{\gamma}\left(S^{2 n+1}\right)$ are introduced in $\S 2.4$. By using the Cayley transform between the Heisenberg group $\mathbb{H}^{n}$ and the CR sphere $S^{2 n+1}$ we prove that there is an explicit correspondence between the weak solutions of $(\mathbf{F Y H})_{\gamma}$ and $(\mathbf{F Y S})_{\gamma}$, respectively, see Proposition 3.1 (and Remark 3.1 for an alternative proof). Being in the critical case, the energy functional associated to problem $(\mathbf{F Y S})_{\gamma}$ does not satisfy the usual Palais-Smale condition due to the lack of compactness of the embedding $H^{\gamma}\left(S^{2 n+1}\right) \hookrightarrow L^{\frac{2 Q}{Q-2 \gamma}}\left(S^{2 n+1}\right)$. In order to regain some compactness, we establish a CR fractional version of the Ding-Hebey-Vaugon compactness result on the CR sphere $S^{2 n+1}$, see Proposition 3.2. In fact, subgroups of the unitary group $\mathbf{U}(n+1)$ having the form $G=\mathbf{U}\left(n_{1}\right) \times \ldots \times \mathbf{U}\left(n_{k}\right)$ with $n_{1}+\ldots+n_{k}=n+1$ will imply the compactness of the embedding of $G$-invariant functions of $H^{\gamma}\left(S^{2 n+1}\right)$ into $L^{\frac{2 Q}{Q-2 \gamma}}\left(S^{2 n+1}\right)$. Here, we shall explore the compactness result of Maalaoui and Martino [20] combined with an iterative argument of Aubin [1] and the technical assumption $\gamma \in \bigcup_{k=1}^{n}\left[k, \frac{k Q}{Q-1}\right)$; some comments on the necessity of the latter assumption are formulated in Remark 3.2. Now, having such a compactness, the fountain theorem and the principle of symmetric criticality applied to the 
energy functional associated to $(\mathbf{F Y S})_{\gamma}$ will guarantee the existence of a whole sequence of $G$-invariant weak solutions for $(\mathbf{F Y S})_{\gamma}$, so for $(\mathbf{F Y H})_{\gamma}$. The number of $\left[\frac{n+1}{2}\right]$ sequences of sign-changing weak solutions for $(\mathbf{F Y H})_{\gamma}$ with mutually different nodal properties will follow by careful choices of the subgroups $G=\mathbf{U}\left(n_{1}\right) \times \ldots \times \mathbf{U}\left(n_{k}\right)$ of the unitary group $\mathbf{U}(n+1)$ with $n_{1}+\ldots+n_{k}=n+1$, see Proposition 3.3.

Plan of the paper. In Section 2 we recall those notions and results that are indispensable to present our argument (e.g. basic facts about Heisenberg groups, the Cayley transform, spherical/zonal harmonics on $S^{2 n+1}$, fractional Sobolev spaces on $S^{2 n+1}$ and $\mathbb{H}^{n}$ ). Section 3 is devoted to the proof of Theorem 1.1; in $\S 3.1$ we prove the equivalence between the weak solutions of problems $(\mathbf{F Y S})_{\gamma}$ and $(\mathbf{F Y H})_{\gamma}$; in $\S 3.2$ we establish the compactness result on the CR fractional setting for $S^{2 n+1}$; in $\S 3.3$ we treat the group-theoretical aspects of our problem concerning the choice of the subgroups $G=\mathbf{U}\left(n_{1}\right) \times \ldots \times \mathbf{U}\left(n_{k}\right)$ of the unitary group $\mathbf{U}(n+1)$ which is needed to produce $\left[\frac{n+1}{2}\right]$ sequences of sign-changing weak solutions for $(\mathbf{F Y H})_{\gamma}$ with different nodal properties. Finally, in $\S 3.4$ we assemble the aforementioned pieces in order to conclude the proof of Theorem 1.1.

\section{Preliminaries}

In order the paper to be self-contained, we recall in this section some basic notions from [5], [13], [14], [15] and [23] which are indispensable in the sequel.

2.1. Heisenberg groups. An element in the Heisenberg group $\mathbb{H}^{n}$ is denoted by $(x, y, t)$, where $x=\left(x_{1}, \ldots, x_{n}\right) \in \mathbb{R}^{n}, y=\left(y_{1}, \ldots, y_{n}\right) \in \mathbb{R}^{n}, t \in \mathbb{R}$, and we identify the pair $(x, y)$ with $z \in \mathbb{C}^{n}$ having coordinates $z_{j}=x_{j}+i y_{j}$ for all $j=1, \ldots, n$. The correspondence with its Lie algebra via the exponential coordinates induces the group law

$$
(z, t) \star\left(z^{\prime}, t^{\prime}\right)=\left(z+z^{\prime}, t+t^{\prime}+2 \operatorname{Im} z \cdot \overline{z^{\prime}}\right), \quad \forall(z, t),\left(z^{\prime}, t^{\prime}\right) \in \mathbb{C}^{n} \times \mathbb{R},
$$

where Im denotes the imaginary part of a complex number and $z \cdot \overline{z^{\prime}}=\sum_{j=1}^{n} z_{j} \overline{z_{j}^{\prime}}$ is the

Hermitian inner product. In these coordinates the neutral element of $\mathbb{H}^{n}$ is $0_{\mathbb{H}^{n}}=\left(0_{\mathbb{C}^{n}}, 0\right)$ and the inverse $(z, t)^{-1}$ of the element $(z, t)$ is $(-z,-t)$. Note that $(x, y, t)=(z, t)$ forms a real coordinate system for $\mathbb{H}^{n}$ and the system of vector fields given as differential operators

$$
X_{j}=\frac{\partial}{\partial x_{j}}+2 y_{j} \frac{\partial}{\partial t}, \quad Y_{j}=\frac{\partial}{\partial y_{j}}-2 x_{j} \frac{\partial}{\partial t}, \quad j \in\{1, \ldots n\}, \quad T=\frac{\partial}{\partial t},
$$

forms a basis of the left-invariant vector fields on $\mathbb{H}^{n}$. The vectors $X_{j}, Y_{j}, j \in\{1, \ldots, n\}$ form the basis of the horizontal bundle. Let

$$
N(z, t)=\left(|z|^{4}+t^{2}\right)^{\frac{1}{4}}
$$

be the homogeneous gauge norm on $\mathbb{H}^{n}$ and $d_{K C}: \mathbb{H}^{n} \times \mathbb{H}^{n} \rightarrow \mathbb{R}$ be the Korányi-Cygan metric given by

$$
d_{K C}\left((z, t),\left(z^{\prime}, t^{\prime}\right)\right)=N\left(\left(z^{\prime}, t^{\prime}\right)^{-1} \star(z, t)\right)=\left(\left|z-z^{\prime}\right|^{4}+\left(t-t^{\prime}-2 \operatorname{Im} z \cdot \overline{z^{\prime}}\right)^{2}\right)^{\frac{1}{4}} .
$$

The Lebesgue measure of $\mathbb{R}^{2 n+1}$ will be the Haar measure on $\mathbb{H}^{n}$ (uniquely defined up to a positive multiplicative constant).

\subsection{Cayley transform. Let}

$$
S^{2 n+1}=\left\{\zeta=\left(\zeta_{1}, \ldots, \zeta_{n+1}\right) \in \mathbb{C}^{n+1}:\langle\zeta, \bar{\zeta}\rangle=1\right\}
$$

be the unit sphere in $\mathbb{C}^{n+1}$, where $\langle\zeta, \bar{\eta}\rangle=\sum_{j=1}^{n+1} \zeta_{j} \overline{\eta_{j}}$. The distance function on $S^{2 n+1}$ is given by

$$
d_{S}(\zeta, \eta)=\sqrt{2|1-\langle\zeta, \bar{\eta}\rangle|}, \quad \zeta, \eta \in S^{2 n+1} .
$$


The Cayley transform $\mathcal{C}: \mathbb{H}^{n} \rightarrow S^{2 n+1} \backslash\{(0, \ldots, 0,-1)\}$ is defined by

$$
\mathcal{C}(z, t)=\left(\frac{2 z}{1+|z|^{2}+i t}, \frac{1-|z|^{2}-i t}{1+|z|^{2}+i t}\right)
$$

whose Jacobian determinant is given by

$$
\operatorname{Jac}_{\mathcal{C}}(z, t)=\frac{2^{2 n+1}}{\left(\left(1+|z|^{2}\right)^{2}+t^{2}\right)^{n+1}}, \quad(z, t) \in \mathbb{H}^{n}
$$

Accordingly, for any integrable function $f: S^{2 n+1} \rightarrow \mathbb{R}$, we have

$$
\int_{S^{2 n+1}} f(\eta) \mathrm{d} \eta=\int_{\mathbb{H}^{n}} f(\mathcal{C}(z, t)) \operatorname{Jac}_{\mathcal{C}}(z, t) \mathrm{d} z \mathrm{~d} t .
$$

If $w=(z, t), v=\left(z^{\prime}, t^{\prime}\right)$ and $\zeta=\mathcal{C}(w), \eta=\mathcal{C}(v)$, one has that

$$
d_{S}(\zeta, \eta)=d_{K C}(w, v)\left(\frac{4}{\left(\left(1+|z|^{2}\right)^{2}+t^{2}\right)}\right)^{\frac{1}{4}}\left(\frac{4}{\left(\left(1+\left|z^{\prime}\right|^{2}\right)^{2}+\left(t^{\prime}\right)^{2}\right)}\right)^{\frac{1}{4}} .
$$

2.3. Spherical/zonal harmonics on $S^{2 n+1}$. The Hilbert space $L^{2}\left(S^{2 n+1}\right)$, endowed by the inner product

$$
(U, V)=\int_{S^{2 n+1}} U \bar{V} \mathrm{~d} \eta
$$

can be decomposed into $\mathbf{U}(n+1)$-irreducible components as

$$
L^{2}\left(S^{2 n+1}\right)=\bigoplus_{j, k \geq 0} \mathcal{H}_{j, k}
$$

where $\mathcal{H}_{j, k}$ denotes the space of harmonic polynomials $p(z, \bar{z})$ on $\mathbb{C}^{n+1}$ restricted to $S^{2 n+1}$ that are homogeneous of degree $j$ and $k$ in the variables $z$ and $\bar{z}$, respectively. We notice that the dimension of $\mathcal{H}_{j, k}$ is

$$
m_{j, k}=\frac{(j+n-1) !(k+n-1) !(j+k+n)}{n !(n-1) ! j ! k !} .
$$

Moreover, if $\left\{Y_{j, k}^{l}\right\}_{l=\overline{1, m_{j, k}}}$ is an orthonormal basis of $\mathcal{H}_{j, k}$, then the zonal harmonics are defined by

$$
\Phi_{j, k}(\zeta, \eta)=\sum_{l=1}^{m_{j, k}} Y_{j, k}^{l}(\zeta) \overline{Y_{j, k}^{l}(\eta)}, \quad \zeta, \eta \in S^{2 n+1} .
$$

We recall that $\Phi_{j, k}$ can be represented as

$$
\Phi_{j, k}(\zeta, \eta)=\frac{(\max \{j, k\}+n-1) !(j+k+n)}{\omega_{2 n+1} n !(\max \{j, k\}) !}\langle\zeta, \bar{\eta}\rangle^{|j-k|} P_{k}^{(n-1,|j-k|)}\left(2\langle\zeta, \bar{\eta}\rangle^{2}-1\right),
$$

where $P_{k}^{(n, l)}$ denotes the Jacobi polynomials and $\omega_{2 n+1}$ is the usual measure of $S^{2 n+1}$.

2.4. Fractional Sobolev spaces on $S^{2 n+1}$ and $\mathbb{H}^{n}$. The usual sub-Laplacian on $\mathbb{H}^{n}$ is defined as

$$
\mathcal{L}=-\frac{1}{4} \sum_{j=1}^{n}\left(X_{j}^{2}+Y_{j}^{2}\right)
$$

If we introduce the differential operators

$$
T_{j}=\frac{\partial}{\partial \eta_{j}}-\bar{\eta}_{j} \sum_{k=1}^{n+1} \eta_{k} \frac{\partial}{\partial \eta_{k}}, \quad \overline{T_{j}}=\frac{\partial}{\partial \overline{\eta_{j}}}-\eta_{j} \sum_{k=1}^{n+1} \overline{\eta_{k}} \frac{\partial}{\partial \overline{\eta_{k}}}, \quad j \in\{1, \ldots, n+1\},
$$


the conformal sub-Laplacian on $S^{2 n+1}$ is given by

$$
\mathcal{D}=-\frac{1}{2} \sum_{j=1}^{n+1}\left(T_{j} \overline{T_{j}}+\overline{T_{j}} T_{j}\right)+\frac{n^{2}}{4}
$$

Note that for every $Y_{j, k} \in \mathcal{H}_{j, k}$ one has

$$
\mathcal{D} Y_{j, k}=\lambda_{j} \lambda_{k} Y_{j, k}
$$

where $\lambda_{j}=j+\frac{n}{2}$.

Let $0<\gamma<\frac{Q}{2}=n+1$ be fixed. Given $U \in L^{2}\left(S^{2 n+1}\right)$, its Fourier representation is

$$
U=\sum_{j, k \geq 0} \sum_{l=1}^{m_{j, k}} c_{j, k}^{l}(U) Y_{j, k}^{l}
$$

with Fourier coefficients $c_{j, k}^{l}(U)=\int_{S^{2 n+1}} U Y_{j, k}^{l} \mathrm{~d} \eta$. Accordingly, we may define

$$
\mathcal{D}^{\frac{\gamma}{2}} U=\sum_{j, k \geq 0} \sum_{l=1}^{m_{j, k}}\left(\lambda_{j} \lambda_{k}\right)^{\frac{\gamma}{2}} c_{j, k}^{l}(U) Y_{j, k}^{l}
$$

The fractional Sobolev space over $S^{2 n+1}$ is defined as

$$
H^{\gamma}\left(S^{2 n+1}\right)=W^{\gamma, 2}\left(S^{2 n+1}\right)=\left\{U \in L^{2}\left(S^{2 n+1}\right): \mathcal{D}^{\frac{\gamma}{2}} U \in L^{2}\left(S^{2 n+1}\right)\right\},
$$

endowed with the inner product and norm

$$
(U, V)_{H^{\gamma}}=\int_{S^{2 n+1}} \mathcal{D}^{\frac{\gamma}{2}} U \overline{\mathcal{D}^{\frac{\gamma}{2}} V} \mathrm{~d} \eta \text { and }\|U\|_{H^{\gamma}}=(U, U)_{H^{\gamma}}^{\frac{1}{2}}=\left(\sum_{j, k \geq 0} \sum_{l=1}^{m_{j, k}}\left(\lambda_{j} \lambda_{k}\right)^{\gamma}\left|c_{j, k}^{l}(U)\right|^{2}\right)^{\frac{1}{2}} .
$$

The norm $\|\cdot\|_{H^{\gamma}}$ is equivalent to the norm coming from the inner product

$$
(U, V)_{\gamma}=\sum_{j, k \geq 0} \sum_{l=1}^{m_{j, k}} \lambda_{j}(\gamma) \lambda_{k}(\gamma) c_{j, k}^{l}(U) \overline{c_{j, k}^{l}(V)}
$$

where

$$
\lambda_{j}(\gamma)=\frac{\Gamma\left(\frac{Q+2 \gamma}{4}+j\right)}{\Gamma\left(\frac{Q-2 \gamma}{4}+j\right)}, \quad j \in \mathbb{N}_{0}=\{0,1,2, \ldots\} ;
$$

indeed, by asymptotic approximation of the Gamma function $\Gamma$, one has $\lambda_{j}(\gamma) \sim j^{\gamma}$.

The intertwining operator $\mathcal{A}_{\gamma}$ of order $2 \gamma$ on $S^{2 n+1}$ is given by

$$
\operatorname{Jac}_{\tau}^{\frac{Q+2 \gamma}{2 Q}}\left(\mathcal{A}_{\gamma} U\right) \circ \tau=\mathcal{A}_{\gamma}\left(\operatorname{Jac}_{\tau}^{\frac{Q-2 \gamma}{2 Q}}(U \circ \tau)\right) \text { for all } \tau \in \operatorname{Aut}\left(S^{2 n+1}\right), U \in C^{\infty}\left(S^{2 n+1}\right),
$$

where $\operatorname{Aut}\left(S^{2 n+1}\right)$ and $\mathrm{Jac}_{\tau}$ denote the group of automorphisms on $S^{2 n+1}$ and the Jacobian of $\tau \in \operatorname{Aut}\left(S^{2 n+1}\right)$, respectively. In fact, the latter definition can be extended to every $U \in H^{\gamma}\left(S^{2 n+1}\right)$. Note that $\mathcal{A}_{\gamma}$ may by characterized (up to a constant) by its action on $\mathcal{H}_{j, k}$ as

$$
\mathcal{A}_{\gamma} Y_{j, k}=\lambda_{j}(\gamma) \lambda_{k}(\gamma) Y_{j, k}, \quad Y_{j, k} \in \mathcal{H}_{j, k}
$$

Therefore,

$$
(U, V)_{\gamma}=\int_{S^{2 n+1}} \bar{V} \mathcal{A}_{\gamma} U \mathrm{~d} \eta
$$


In particular, $\lambda_{j}(1)=\lambda_{j}$ for every $j \in \mathbb{N}_{0}$ and $\mathcal{A}_{1}=\mathcal{D}$. Moreover, according to Frank and Lieb [14], for every real-valued function $U \in H^{\gamma}\left(S^{2 n+1}\right)$, one has the sharp fractional Sobolev inequality on the CR sphere $S^{2 n+1}$, i.e.,

$$
\left(\int_{S^{2 n+1}}|U(\eta)|^{\frac{2 Q}{Q-2 \gamma}} \mathrm{d} \eta\right)^{\frac{Q-2 \gamma}{Q}} \leq C(\gamma, n) \int_{S^{2 n+1}} U(\eta) \mathcal{A}_{\gamma} U(\eta) \mathrm{d} \eta
$$

where

$$
C(\gamma, n)=\frac{\Gamma\left(\frac{n+1-\gamma}{2}\right)^{2}}{\Gamma\left(\frac{n+1+\gamma}{2}\right)^{2}} \omega_{2 n+1}^{-\frac{\gamma}{n+1}} .
$$

The CR fractional sub-Laplacian operator on $\mathbb{H}^{n}$ is defined by

$$
\mathcal{L}_{\gamma}=|2 T|^{\gamma} \frac{\Gamma\left(\mathcal{L}|2 T|^{-1}+\frac{1+\gamma}{2}\right)}{\Gamma\left(\mathcal{L}|2 T|^{-1}+\frac{1-\gamma}{2}\right)} .
$$

Direct computation shows that $\mathcal{L}_{1}=\mathcal{L}, \mathcal{L}_{2}=\mathcal{L}^{2}-|T|^{2}$. Moreover, the relationship between $\mathcal{L}_{\gamma}$ and $\mathcal{A}_{\gamma}$ is given by

$$
\mathcal{L}_{\gamma}\left(\left(2 \operatorname{Jac}_{\mathcal{C}}\right)^{\frac{Q-2 \gamma}{2 Q}}(U \circ \mathcal{C})\right)=\left(2 \operatorname{Jac}_{\mathcal{C}}\right)^{\frac{Q+2 \gamma}{2 Q}}\left(\mathcal{A}_{\gamma} U\right) \circ \mathcal{C}, \quad \forall U \in H^{\gamma}\left(S^{2 n+1}\right) .
$$

The fractional Sobolev space over $\mathbb{H}^{n}$ is defined by

$$
D^{\gamma}\left(\mathbb{H}^{n}\right)=\left\{u \in L^{\frac{2 Q}{Q-2 \gamma}}\left(\mathbb{H}^{n}\right): a_{\gamma}[u]<+\infty\right\},
$$

where the quadratic form $a_{\gamma}$ is associated to the operator $\mathcal{L}_{\gamma}$, i.e.,

$$
a_{\gamma}[u]=\int_{\mathbb{H}^{n}} \bar{u} \mathcal{L}_{\gamma} u \mathrm{~d} z \mathrm{~d} t .
$$

The form $a_{\gamma}$ can be equivalently represented by means of spectral decomposition, see [15, p. 126].

\section{Proof of Main Theorem}

3.1. Equivalent critical problems on $\mathbb{H}^{n}$ and $S^{2 n+1}$. Let $\gamma \in(0, n+1)$ be fixed. We consider the fractional Yamabe problem on the CR sphere, i.e.,

$$
\left\{\begin{array}{l}
\mathcal{A}_{\gamma} U=\mid U^{\frac{4 \gamma}{Q-2 \gamma}} U \text { on } S^{2 n+1} \\
U \in H^{\gamma}\left(S^{2 n+1}\right)
\end{array}\right.
$$

Hereafter, we are considering real-valued functions both in $H^{\gamma}\left(S^{2 n+1}\right)$ and $D^{\gamma}\left(\mathbb{H}^{n}\right)$, respectively. The main result of this subsection constitutes the bridge between $(\mathbf{F Y S})_{\gamma}$ and $(\mathbf{F Y H})_{\gamma}$ as follows:

Proposition 3.1. Let $0<\gamma<\frac{Q}{2}=n+1$. Then $U \in H^{\gamma}\left(S^{2 n+1}\right)$ is a weak solution of $(\mathbf{F Y S})_{\gamma}$ if and only if $u=\left(2 \mathrm{Jac}_{\mathcal{C}}\right)^{\frac{Q-2 \gamma}{2 Q}} U \circ \mathcal{C} \in D^{\gamma}\left(\mathbb{H}^{n}\right)$ is a weak solution of $(\mathbf{F Y H})_{\gamma}$.

Proof. We first prove the following

$\underline{\text { Claim: }}$ Let $U: S^{2 n+1} \rightarrow \mathbb{R}$ and $u: \mathbb{H}^{n} \rightarrow \mathbb{R}$ be two functions such that $u=\operatorname{Jac}_{\mathcal{C}}^{\frac{Q-2 \gamma}{2 Q}} U \circ \mathcal{C}$. Then $U \in H^{\gamma}\left(S^{2 n+1}\right)$ if and only if $u \in D^{\gamma}\left(\mathbb{H}^{n}\right)$.

Fix $U \in H^{\gamma}\left(S^{2 n+1}\right)$; we shall prove first that $(z, t) \mapsto u(z, t)=\operatorname{Jac}_{\mathcal{C}}(z, t)^{\frac{Q-2 \gamma}{2 Q}} U(\mathcal{C}(z, t))$ belongs to $D^{\gamma}\left(\mathbb{H}^{n}\right)$. By (2.1) one has

$$
\begin{aligned}
\int_{\mathbb{H}^{n}}|u(z, t)|^{\frac{2 Q}{Q-2 \gamma}} \mathrm{d} z \mathrm{~d} t & =\int_{\mathbb{H}^{n}} \operatorname{Jac}_{\mathcal{C}}(z, t)|U(\mathcal{C}(z, t))|^{\frac{2 Q}{Q-2 \gamma}} \mathrm{d} z \mathrm{~d} t \\
& =\int_{S^{2 n+1}}|U(\eta)|^{\frac{2 Q}{Q-2 \gamma}} \mathrm{d} \eta .
\end{aligned}
$$


Furthermore, by the fractional Sobolev inequality (2.7) and relation (2.5), one has that

$$
\begin{aligned}
\left(\int_{S^{2 n+1}}|U(\eta)|^{\frac{2 Q}{Q-2 \gamma}} \mathrm{d} \eta\right)^{\frac{Q-2 \gamma}{Q}} & \leq C(\gamma, n) \int_{S^{2 n+1}} U(\eta) \mathcal{A}_{\gamma} U(\eta) \mathrm{d} \eta \\
& =C(\gamma, n) \sum_{j, k \geq 0} \sum_{l=1}^{m_{j, k}} \lambda_{j}(\gamma) \lambda_{k}(\gamma)\left|c_{j, k}^{l}(U)\right|^{2} \\
& \leq C^{\prime}(\gamma, n)\|U\|_{H^{\gamma}}^{2} \\
& <+\infty
\end{aligned}
$$

where $C^{\prime}(\gamma, n)=C_{\gamma} C(\gamma, n)$ and $C_{\gamma}>0$ is such that $(V, V)_{\gamma} \leq C_{\gamma}\|V\|_{H^{\gamma}}^{2}$ for every $V \in H^{\gamma}\left(S^{2 n+1}\right)$; thus $u \in L^{\frac{2 Q}{Q-2 \gamma}}\left(\mathbb{H}^{n}\right)$. Moreover, by (2.8) and (2.1) one has

$$
\begin{aligned}
a_{\gamma}[u] & =\int_{\mathbb{H}^{n}} u \mathcal{L}_{\gamma} u \mathrm{~d} z \mathrm{~d} t= \\
& =2^{\alpha^{\prime}} \int_{\mathbb{H}^{n}} \operatorname{Jac}_{\mathcal{C}}(z, t)^{\frac{Q-2 \gamma}{2 Q}} U(\mathcal{C}(z, t)) \mathcal{L}_{\gamma}\left(\left(2 \operatorname{Jac}_{\mathcal{C}}(z, t)\right)^{\frac{Q-2 \gamma}{2 Q}} U(\mathcal{C}(z, t))\right) \mathrm{d} z \mathrm{~d} t \\
& =2^{\alpha^{\prime}} \int_{\mathbb{H}^{n}} \operatorname{Jac}_{\mathcal{C}}(z, t)^{\frac{Q-2 \gamma}{2 Q}} U(\mathcal{C}(z, t))\left(2 \operatorname{Jac}_{\mathcal{C}}(z, t)\right)^{\frac{Q+2 \gamma}{2 Q}}\left(\mathcal{A}_{\gamma} U\right)(\mathcal{C}(z, t)) \mathrm{d} z \mathrm{~d} t \\
& =2^{\alpha^{\prime \prime}} \int_{\mathbb{H}^{n}} U(\mathcal{C}(z, t))\left(\mathcal{A}_{\gamma} U\right)(\mathcal{C}(z, t)) \operatorname{Jac}_{\mathcal{C}}(z, t) \mathrm{d} z \mathrm{~d} t \\
& =2^{\alpha^{\prime \prime}} \int_{S^{2 n+1}} U(\eta) \mathcal{A}_{\gamma} U(\eta) \mathrm{d} \eta \\
& <+\infty
\end{aligned}
$$

where $\alpha^{\prime}=-\frac{Q-2 \gamma}{2 Q}$ and $\alpha^{\prime \prime}=\alpha^{\prime}+\frac{Q+2 \gamma}{2 Q}=\frac{2 \gamma}{Q}$. Therefore, $u \in D^{\gamma}\left(\mathbb{H}^{n}\right)$.

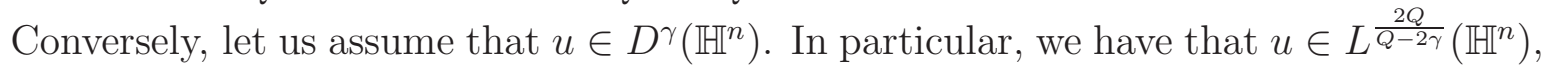
thus by relation (3.1) it turns out that $U \in L^{\frac{2 Q}{Q-2 \gamma}}\left(S^{2 n+1}\right)$; therefore, $U \in L^{2}\left(S^{2 n+1}\right)$. Furthermore, by (3.2) we also have that

$$
\int_{S^{2 n+1}} U(\eta) \mathcal{A}_{\gamma} U(\eta) \mathrm{d} \eta=2^{-\alpha^{\prime \prime}} a_{\gamma}[u]<+\infty
$$

i.e., $U \in H^{\gamma}\left(S^{2 n+1}\right)$, which concludes the proof of Claim.

Let $U \in H^{\gamma}\left(S^{2 n+1}\right)$ be a weak solution of $(\mathbf{F Y S})_{\gamma}$; then we have

$$
\int_{S^{2 n+1}} \mathcal{A}_{\gamma} U V \mathrm{~d} \eta=\int_{S^{2 n+1}}|U|^{\frac{4 \gamma}{Q-2 \gamma}} U V \mathrm{~d} \eta \quad \text { for every } V \in H^{\gamma}\left(S^{2 n+1}\right) .
$$

Let $v \in D^{\gamma}\left(\mathbb{H}^{n}\right)$ be arbitrarily fixed and define $V=\left(\operatorname{Jac}_{\mathcal{C}} \circ \mathcal{C}^{-1}\right)^{\frac{2 \gamma-Q}{2 Q}} v \circ \mathcal{C}^{-1}$. Since $v=\operatorname{Jac}_{\mathcal{C}}{ }^{\frac{Q-2 \gamma}{2 Q}} V \circ \mathcal{C}$, by the Claim we have that $V \in H^{\gamma}\left(S^{2 n+1}\right)$. Accordingly, the function $V$ can be used as a test-function in (3.3), obtaining

$$
\int_{S^{2 n+1}} \mathcal{A}_{\gamma} U\left(\operatorname{Jac}_{\mathcal{C}} \circ \mathcal{C}^{-1}\right)^{\frac{2 \gamma-Q}{2 Q}} v \circ \mathcal{C}^{-1} \mathrm{~d} \eta=\int_{S^{2 n+1}}|U|^{\frac{4 \gamma}{Q-2 \gamma}} U\left(\operatorname{Jac}_{\mathcal{C}} \circ \mathcal{C}^{-1}\right)^{\frac{2 \gamma-Q}{2 Q}} v \circ \mathcal{C}^{-1} \mathrm{~d} \eta .
$$

By a change of variables, it follows that

$$
\int_{\mathbb{H}^{n}}\left(\mathcal{A}_{\gamma} U \circ \mathcal{C}\right)\left(\operatorname{Jac}_{\mathcal{C}}\right)^{\frac{2 \gamma-Q}{2 Q}+1} v \mathrm{~d} z \mathrm{~d} t=\int_{\mathbb{H}^{n}}|U \circ \mathcal{C}|^{\frac{4 \gamma}{Q-2 \gamma}}(U \circ \mathcal{C})\left(\operatorname{Jac}_{\mathcal{C}}\right)^{\frac{2 \gamma-Q}{2 Q}+1} v \mathrm{~d} z \mathrm{~d} t .
$$

This relation and (2.8) imply that

$$
2^{-\frac{Q+2 \gamma}{2 Q}} \int_{\mathbb{H}^{n}} \mathcal{L}_{\gamma}\left(\left(2 \mathrm{Jac}_{\mathcal{C}}\right)^{\frac{Q-2 \gamma}{2 Q}}(U \circ \mathcal{C})\right) v \mathrm{~d} z \mathrm{~d} t=\int_{\mathbb{H}^{n}}|U \circ \mathcal{C}|^{\frac{4 \gamma}{Q-2 \gamma}} U \circ \mathcal{C}\left(\operatorname{Jac}_{\mathcal{C}}\right)^{\frac{2 \gamma+Q}{2 Q}} v \mathrm{~d} z \mathrm{~d} t
$$


Since $u=\left(2 \mathrm{Jac}_{\mathcal{C}}\right)^{\frac{Q-2 \gamma}{2 Q}} U \circ \mathcal{C}$, the latter equality is equivalent to

$$
\int_{\mathbb{H}^{n}} \mathcal{L}_{\gamma} u v \mathrm{~d} z \mathrm{~d} t=\int_{\mathbb{H}^{n}}|u|^{\frac{4 \gamma}{Q-2 \gamma}} u v \mathrm{~d} z \mathrm{~d} t
$$

which means precisely that $u \in D^{\gamma}\left(\mathbb{H}^{n}\right)$ is a weak solution of $(\mathbf{F Y H})_{\gamma}$. The converse argument works in a similar way.

Remark 3.1. One can provide an alternative proof to Proposition 3.1 by exploring the explicit form of the fundamental solution of $\mathcal{L}_{\gamma}$; a similar approach is due to Bartsch, Schneider and Weth [3] for the polyharmonic operator $(-\Delta)^{m}$ in $\mathbb{R}^{N}$, where $m \in \mathbb{N}$ and $N>2 m$. For completeness, we sketch the proof.

We recall that the fundamental solution of $\mathcal{L}_{\gamma}$ is

$$
\mathcal{L}_{\gamma}^{-1}\left((z, t),\left(z^{\prime}, t^{\prime}\right)\right)=\frac{c_{\gamma}}{2} d_{K C}^{2 \gamma-Q}\left((z, t),\left(z^{\prime}, t^{\prime}\right)\right)
$$

where

$$
c_{\gamma}=\frac{2^{n-\gamma} \Gamma\left(\frac{Q-2 \gamma}{4}\right)^{2}}{\pi^{n+1} \Gamma(\gamma)},
$$

see Branson, Fontana and Morpurgo [5, p. 21]. For every $\psi \in L^{\frac{2 Q}{Q+2 \gamma}}\left(S^{2 n+1}\right)$ we introduce the function

$$
\left[\mathcal{K}_{\gamma} \psi\right](\zeta)=c_{\gamma} \int_{S^{2 n+1}} \psi(\eta)|1-\langle\zeta, \bar{\eta}\rangle|^{\frac{2 \gamma-Q}{2}} \mathrm{~d} \eta .
$$

One can prove that $\mathcal{K}_{\gamma} \psi \in H^{\gamma}\left(S^{2 n+1}\right)$ for every $\psi \in L^{\frac{2 Q}{Q+2 \gamma}}\left(S^{2 n+1}\right)$. Moreover, the FunkHecke theorem on the CR sphere $S^{2 n+1}$ gives

$$
\left[\mathcal{K}_{\gamma} Y_{j, k}\right](\zeta)=\frac{2^{\frac{Q}{2}-\gamma}}{\lambda_{j}(\gamma) \lambda_{k}(\gamma)} Y_{j, k}(\zeta),
$$

see Frank and Lieb [14, Corollary 5.3]. Thus, a direct computation yields that

$$
\left(\mathcal{K}_{\gamma} \psi, V\right)_{\gamma}=2^{\frac{Q}{2}-\gamma} \int_{S^{2 n+1}} \psi V \mathrm{~d} \eta \quad \text { for every } V \in H^{\gamma}\left(S^{2 n+1}\right) .
$$

Note that if $U \in H^{\gamma}\left(S^{2 n+1}\right)$ is a weak solution of $(\mathbf{F Y S})_{\gamma}$, the latter relation implies that

$$
\mathcal{K}_{\gamma}\left(|U|^{\frac{4 \gamma}{Q-2 \gamma}} U\right)=2^{\frac{Q}{2}-\gamma} U \text { on } S^{2 n+1} .
$$

Accordingly, by relations (3.6), (3.5), (2.1) and (2.2), it turns out that

$$
\begin{aligned}
u(z, t) & =\left(2 \operatorname{Jac}_{\mathcal{C}}(z, t)\right)^{\frac{Q-2 \gamma}{2 Q}} U(\mathcal{C}(z, t)) \\
& =2^{-\frac{Q}{2}+\gamma}\left(2 \operatorname{Jac}_{\mathcal{C}}(z, t)\right)^{\frac{Q-2 \gamma}{2 Q}} \mathcal{K}_{\gamma}\left(|U(\mathcal{C}(z, t))|^{\frac{4 \gamma}{Q-2 \gamma}} U(\mathcal{C}(z, t))\right) \\
& =\frac{c_{\gamma}}{2} \int_{\mathbb{H}^{n}} d_{K C}^{2 \gamma-Q}\left((z, t),\left(z^{\prime}, t^{\prime}\right)\left|u\left(z^{\prime}, t^{\prime}\right)\right|^{\frac{4 \gamma}{Q-\gamma}} u\left(z^{\prime}, t^{\prime}\right) \mathrm{d} z^{\prime} \mathrm{d} t^{\prime}, \quad(z, t) \in \mathbb{H}^{n} .\right.
\end{aligned}
$$

The latter relation is equivalent to the fact that

$$
u(z, t)=\frac{c_{\gamma}}{2}\left(|u|^{\frac{4 \gamma}{Q-\gamma}} u\right) * d_{K C}^{2 \gamma-Q}((z, t), \cdot), \quad(z, t) \in \mathbb{H}^{n},
$$

where ${ }^{\prime} *$ denotes the (noncommutative) convolution operation on the Heisenberg group $\mathbb{H}^{n}$. By (3.4), a similarly argument as in Folland [12, Theorem 2] gives that $\mathcal{L}_{\gamma} u=|u|^{\frac{4 \gamma}{Q-\gamma}} u$ on $\mathbb{H}^{n}$, which concludes the claim. 
3.2. Compactness. According to Frank and Lieb [14], see also (2.7), the embedding $H^{\gamma}\left(S^{2 n+1}\right) \hookrightarrow L^{\frac{2 Q}{Q-2 \gamma}}\left(S^{2 n+1}\right)$ is continuous, but not compact. This subsection is devoted to regain certain compactness by using suitable group actions on the CR sphere $S^{2 n+1}$.

To complete this purpose, let $n_{j} \in \mathbb{N}, j=1, \ldots, k$, with $n_{1}+\ldots+n_{k}=n+1$. Associated to these numbers, let

$$
G=\mathbf{U}\left(n_{1}\right) \times \ldots \times \mathbf{U}\left(n_{k}\right)
$$

be the subgroup of the unitary group $\mathbf{U}(n+1)=\{g \in \mathbf{O}(2 n+2): g J=J g\}$, where

$$
J=\left[\begin{array}{cc}
0 & I_{\mathbb{R}^{n+1}} \\
-I_{\mathbb{R}^{n+1}} & 0
\end{array}\right]
$$

Let

$$
H_{G}^{\gamma}\left(S^{2 n+1}\right)=\left\{U \in H^{\gamma}\left(S^{2 n+1}\right): g \circ U=U \text { for every } g \in G\right\}
$$

be the subspace of $G$-invariant functions of $H^{\gamma}\left(S^{2 n+1}\right)$, where

$$
(g \circ U)(\eta)=U\left(g^{-1} \eta\right), \quad \eta \in S^{2 n+1} .
$$

It is clear that $H_{G}^{\gamma}\left(S^{2 n+1}\right)$ is an infinite-dimensional closed subspace of $H^{\gamma}\left(S^{2 n+1}\right)$, whenever $k \geq 2$ in the splitting (3.8).

With the above notations in our mind, a Ding-Hebey-Vaugon-type compactness result reads as follows:

Proposition 3.2. Let $\gamma \in \bigcup_{k=1}^{n}\left[k, \frac{k Q}{Q-1}\right)$. The embedding $H_{G}^{\gamma}\left(S^{2 n+1}\right) \hookrightarrow L^{\frac{2 Q}{Q-2 \gamma}}\left(S^{2 n+1}\right)$ is compact, where $G=\mathbf{U}\left(n_{1}\right) \times \ldots \times \mathbf{U}\left(n_{k}\right)$ is any choice with $n_{j} \in \mathbb{N}, j=1, \ldots, k$, and $n_{1}+\ldots+n_{k}=n+1$.

Proof. First, when $G=\mathbf{U}(n+1)$, the space $H_{G}^{\gamma}\left(S^{2 n+1}\right)$ contains precisely the constant functions defined on $S^{2 n+1}$; in this case the proof is trivial.

In the general case, we recall by Maalaoui and Martino [20, Lemma 3.3] that the embedding $W_{G}^{1,2}\left(S^{2 n+1}\right)=H_{G}^{1}\left(S^{2 n+1}\right) \hookrightarrow L^{q}\left(S^{2 n+1}\right)$ is compact for every $1 \leq q<q_{1}^{*}$, where $q_{1}^{*}=\frac{2(Q-1)}{Q-3}$ is the Riemannian critical exponent on the $(Q-1)$-dimensional sphere $S^{2 n+1}$.

By our assumption $\gamma \in \bigcup_{k=1}^{n}\left[k, \frac{k Q}{Q-1}\right)$ we have that $l:=[\gamma] \geq 1$ and

$$
\gamma\left(1-\frac{1}{Q}\right)<l \leq \gamma
$$

The iterative argument developed by Aubin [1, Proposition 2.11], applied for $l$ times, gives that the embedding $W_{G}^{l, 2}\left(S^{2 n+1}\right)=H_{G}^{l}\left(S^{2 n+1}\right) \hookrightarrow L^{q}\left(S^{2 n+1}\right)$ is compact for every $1 \leq q<q_{l}^{*}$, where $q_{l}^{*}=\frac{2(Q-1)}{Q-1-2 l}$. On one hand, since $l \leq \gamma$, we have that $H_{G}^{\gamma}\left(S^{2 n+1}\right)=$ $W_{G}^{\gamma, 2}\left(S^{2 n+1}\right) \subset W_{G}^{l, 2}\left(S^{2 n+1}\right)$. On the other hand, the left hand side of (3.10) is equivalent to $q_{l}^{*}>\frac{2 Q}{Q-2 \gamma}$. Combining these facts, we have the chain of inclusions

$$
H_{G}^{\gamma}\left(S^{2 n+1}\right) \subset W_{G}^{l, 2}\left(S^{2 n+1}\right) \hookrightarrow L^{\frac{2 Q}{Q-2 \gamma}}\left(S^{2 n+1}\right),
$$

where the latter embedding is compact.

Remark 3.2. Our assumption $\gamma \in \bigcup_{k=1}^{n}\left[k, \frac{k Q}{Q-1}\right)$ is important to guarantee the left hand side of (3.10), which in turn, implies that $\frac{2 Q}{Q-2 \gamma}$ is within the range $\left[1, q_{l}^{*}\right)$ where the embedding $W_{G}^{l, 2}\left(S^{2 n+1}\right) \hookrightarrow L^{q}\left(S^{2 n+1}\right)$ is compact, $q \in\left[1, q_{l}^{*}\right)$. We are wondering if this assumption can be removed in order to prove the compactness of the above embedding for the whole spectrum $\left(0, \frac{Q}{2}\right)$ of the parameter $\gamma$. 
3.3. Special group actions. The goal of this subsection is to describe symmetrically different functions belonging to $H^{\gamma}\left(S^{2 n+1}\right)$ via subgroups of the form $G=\mathbf{U}\left(n_{1}\right) \times \ldots \times$ $\mathrm{U}\left(n_{k}\right)$ with $n_{1}+\ldots+n_{k}=n+1$. To handle this problem, we explore a Rubik-cube technique, described in a slightly different manner in Balogh and Kristály [2]; roughly speaking, $n+1$ corresponds to the number of total sides of the cube, while the sides of the cube are certain blocks in the decomposition subgroup $G=\mathbf{U}\left(n_{1}\right) \times \ldots \times \mathbf{U}\left(n_{k}\right)$.

To be more precise, let $n \geq 1$ and for $i \in\left\{1, \ldots,\left[\frac{n+1}{2}\right]\right\}$ we consider the subgroup of the unitary group $\mathbf{U}(n+1)$ as

$$
G_{i}=\left\{\begin{array}{cc}
{\left[\begin{array}{cc}
\mathbf{U}\left(\frac{n+1}{2}\right) & 0 \\
0 & \mathbf{U}\left(\frac{n+1}{2}\right)
\end{array}\right],} & \text { if } n+1=2 i, \\
{\left[\begin{array}{ccc}
\mathbf{U}(i) & 0 & 0 \\
0 & \mathbf{U}(n+1-2 i) & 0 \\
0 & 0 & \mathbf{U}(i)
\end{array}\right],} & \text { if } n+1 \neq 2 i .
\end{array}\right.
$$

It is clear that a particular $G_{i}$ does not act transitively on the sphere $S^{2 n+1}$. However, to recover the transitivity, we shall combine different groups of the type $G_{i}$; for further use, let $\left[G_{i} ; G_{j}\right]$ be the group generated by $G_{i}$ and $G_{j}$.

Lemma 3.1. Let $i, j \in\left\{1, \ldots,\left[\frac{n+1}{2}\right]\right\}$ with $i \neq j$. Then the group $\left[G_{i} ; G_{j}\right]$ acts transitively on the $C R$ sphere $S^{2 n+1}$.

Proof. Without loss of generality, we assume that $j>i$. For further use, let $0_{k}=$ $(0, \ldots, 0) \in \mathbb{C}^{k}=\mathbb{R}^{2 k}, k \in\{1, \ldots, n\}$. Let us fix $\eta=\left(\eta_{1}, \eta_{2}, \eta_{3}\right) \in S^{2 n+1}$ arbitrarily with $\eta_{1}, \eta_{3} \in \mathbb{C}^{j}$ and $\eta_{2} \in \mathbb{C}^{n+1-2 j}$; clearly, $\eta_{2}$ disappears from $\eta$ whenever $2 j=n+1$. Taking into account the fact that $\mathbf{U}(j)$ acts transitively on $S^{2 j-1}$, there are $g_{j}^{1}, g_{j}^{2} \in \mathbf{U}(j)$ such that if $g_{j}=g_{j}^{1} \times I_{\mathbb{C}^{n+1-2 j}} \times g_{j}^{2} \in G_{j}$, then $g_{j} \eta=\left(0_{j-1}, 0,\left|\eta_{1}\right|, \eta_{2},\left|\eta_{3}\right|, 0,0_{j-1}\right)$. Since $j-1 \geq i$, the transitive action of $\mathbf{U}(n+1-2 i)$ on $S^{2 n+1-4 i}$ implies the existence of $g_{i}^{1} \in \mathbf{U}(n+1-2 i)$ such that $g_{i}^{1}\left(0_{j-i-1}, 0,\left|\eta_{1}\right|, \eta_{2},\left|\eta_{3}\right|, 0,0_{j-i-1}\right)=\left(1,0,0_{n-2 i}\right)$. Therefore, if $g_{i}=I_{\mathbb{C}^{i}} \times g_{i}^{1} \times I_{\mathbb{C}^{i}} \in G_{i}$ then $g_{i} g_{j} \eta=\left(0_{i}, 1,0,0_{n-i}\right) \in S^{2 n+1}$.

By repeating the same procedure for another element $\tilde{\eta} \in S^{2 n+1}$, there exists $\tilde{g}_{i} \in G_{i}$ and $\tilde{g}_{j} \in G_{j}$ such that $\tilde{g}_{i} \tilde{g}_{j} \tilde{\eta}=\left(0_{i}, 1,0,0_{n-i}\right) \in S^{2 n+1}$. Accordingly,

$$
\eta=g_{j}^{-1} g_{i}^{-1} \tilde{g}_{i} \tilde{g}_{j} \tilde{\eta}=g_{j}^{-1} \bar{g}_{i} \tilde{g}_{j} \tilde{\eta}
$$

where $\bar{g}_{i}=g_{i}^{-1} \tilde{g}_{i} \in G_{i}$, which concludes the proof.

For every fixed $i \in\left\{1, \ldots,\left[\frac{n+1}{2}\right]\right\}$, we introduce the matrix $A_{i}$ as

$$
A_{i}=\left\{\begin{array}{cc}
{\left[\begin{array}{cc}
0 & I_{\mathbb{C}^{\frac{n+1}{2}}} \\
I_{\mathbb{C}^{\frac{n+1}{2}}} & 0
\end{array}\right],} & \text { if } n+1=2 i, \\
{\left[\begin{array}{ccc}
0 & 0 & I_{\mathbb{C}^{i}} \\
0 & I_{\mathbb{C}^{n+1-2 i}} & 0 \\
I_{\mathbb{C}^{i}} & 0 & 0
\end{array}\right],} & \text { if } n+1 \neq 2 i .
\end{array}\right.
$$

The following construction is inspired by Bartsch and Willem [4]. Since one has $A_{i} \in$ $\mathrm{U}(n+1) \backslash G_{i}, A_{i}^{2}=I_{\mathbb{C}^{n+1}}$ and $A_{i} G_{i}=G_{i} A_{i}$, the group generated by $G_{i}$ and $A_{i}$ is 


$$
\begin{aligned}
& \hat{G}_{i}=\left[G_{i} ; A_{i}\right]=G_{i} \cup A_{i} G_{i} \text {, i.e., }
\end{aligned}
$$

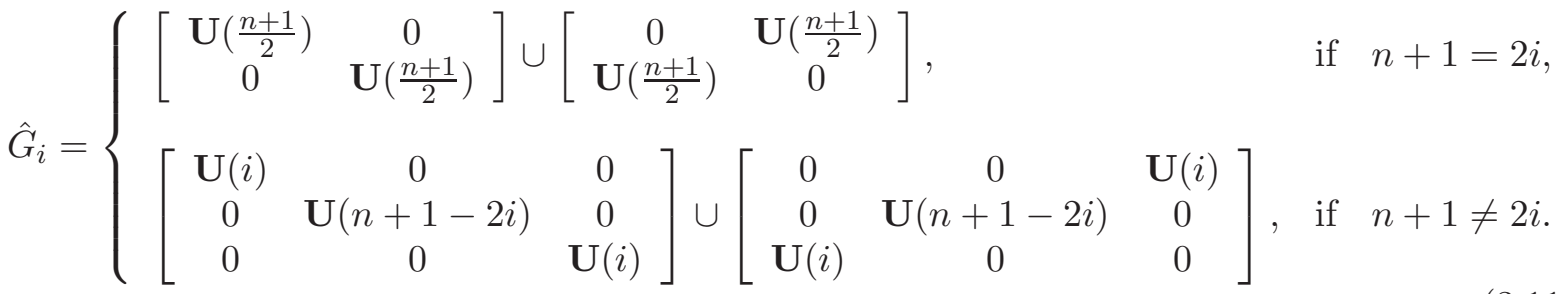

In fact, in $\hat{G}_{i}$ there are only two types of elements: either of the form $g \in G_{i}$, or $A_{i} g \in \hat{G}_{i} \backslash G_{i}$ (with $g \in G_{i}$ ), respectively.

The action $\hat{G}_{i} \circledast H^{\gamma}\left(S^{2 n+1}\right) \mapsto H^{\gamma}\left(S^{2 n+1}\right)$ of the group $\hat{G}_{i}$ on $H^{\gamma}\left(S^{2 n+1}\right)$ is defined by

$$
(\hat{g} \circledast U)(\eta)=\left\{\begin{array}{cl}
U\left(g^{-1} \eta\right), & \text { if } \quad \hat{g}=g \in G_{i}, \\
-U\left(g^{-1} A_{i}^{-1} \eta\right), & \text { if } \quad \hat{g}=A_{i} g \in \hat{G}_{i} \backslash G_{i},
\end{array}\right.
$$

for every $\hat{g} \in \hat{G}_{i}, U \in H^{\gamma}\left(S^{2 n+1}\right)$ and $\eta \in S^{2 n+1}$. We notice that this action is welldefined, continuous and linear. Similarly as in (3.9), we introduce the space of $G_{i}$-invariant functions of $H^{\gamma}\left(S^{2 n+1}\right)$ as

$$
H_{G_{i}}^{\gamma}\left(S^{2 n+1}\right)=\left\{U \in H^{\gamma}\left(S^{2 n+1}\right): g \circ U=U \text { for every } g \in G_{i}\right\},
$$

where the action ' $\mathrm{o}^{\prime}$ corresponds to the first relation in (3.12). Furthermore, let

$$
H_{\hat{G}_{i}}^{\gamma}\left(S^{2 n+1}\right)=\left\{U \in H^{\gamma}\left(S^{2 n+1}\right): \hat{g} \circledast U=U \quad \text { for every } \hat{g} \in \hat{G}_{i}\right\}
$$

be the space of $\hat{G}_{i}$-invariant functions of $H^{\gamma}\left(S^{2 n+1}\right)$.

The following result summarizes the constructions in this subsection.

Proposition 3.3. Let $\gamma \in \bigcup_{k=1}^{n}\left[k, \frac{k Q}{Q-1}\right)$, and fix $i, j \in\left\{1, \ldots,\left[\frac{n+1}{2}\right]\right\}$ such that $i \neq j$. The following statements hold:

(i) The embedding $H_{\hat{G}_{i}}^{\gamma}\left(S^{2 n+1}\right) \hookrightarrow L^{\frac{2 Q}{Q-2 \gamma}}\left(S^{2 n+1}\right)$ is compact;

(ii) $H_{G_{i}}^{\gamma}\left(S^{2 n+1}\right) \cap H_{G_{j}}^{\gamma}\left(S^{2 n+1}\right)=\left\{\right.$ constant functions on $\left.S^{2 n+1}\right\}$;

(iii) $H_{\hat{G}_{i}}^{\gamma}\left(S^{2 n+1}\right) \cap H_{\hat{G}_{j}}^{\gamma}\left(S^{2 n+1}\right)=\{0\}$.

Proof. (i) It is clear that $H_{\hat{G}_{i}}^{\gamma}\left(S^{2 n+1}\right) \subset H_{G_{i}}^{\gamma}\left(S^{2 n+1}\right)$. Moreover, by Proposition 3.2 we have that the embedding $H_{G_{i}}^{\gamma}\left(S^{2 n+1}\right) \hookrightarrow L^{\frac{2 Q}{Q-2 \gamma}}\left(S^{2 n+1}\right)$ is compact.

(ii) Let us fix $U \in H_{G_{i}}^{\gamma}\left(S^{2 n+1}\right) \cap H_{G_{j}}^{\gamma}\left(S^{2 n+1}\right)$. Since $U$ is both $G_{i^{-}}$and $G_{j^{-}}$invariant, it is also $\left[G_{i} ; G_{j}\right]$-invariant, i.e., $U(g \eta)=U(\eta)$ for every $g \in\left[G_{i} ; G_{j}\right]$ and $\eta \in S^{2 n+1}$. According to Lemma 3.1, the group $\left[G_{i} ; G_{j}\right]$ acts transitively on the CR sphere $S^{2 n+1}$, i.e., the orbit of every element $\eta \in S^{2 n+1}$ by the group $\left[G_{i} ; G_{j}\right]$ is the whole sphere $S^{2 n+1}$. Thus, $U$ is a constant function.

(iii) Let $U \in H_{\hat{G}_{i}}^{\gamma}\left(S^{2 n+1}\right) \cap H_{\hat{G}_{j}}^{\gamma}\left(S^{2 n+1}\right)$. On one hand, by (ii), we first have that $U$ is constant. On the other hand, the second relation from (3.12) implies that $U(\eta)=-U\left(A_{i} \eta\right)$ for every $\eta \in S^{2 n+1}$. Therefore, we necessarily have that $U=0$.

3.4. Proof of Theorem 1.1. We associate to problem $(\mathbf{F Y S})_{\gamma}$ the energy functional $E: H^{\gamma}\left(S^{2 n+1}\right) \rightarrow \mathbb{R}$ defined by

$$
E(U)=\frac{1}{2} \int_{S^{2 n+1}} U \mathcal{A}_{\gamma} U \mathrm{~d} \eta-\frac{Q-2 \gamma}{2 Q} \int_{S^{2 n+1}}|U|^{\frac{2 Q}{Q-2 \gamma}} \mathrm{d} \eta, \quad U \in H^{\gamma}\left(S^{2 n+1}\right) .
$$


Due to (2.7), the functional $E$ is well-defined, belonging to $C^{1}\left(H^{\gamma}\left(S^{2 n+1}\right), \mathbb{R}\right)$. Moreover, $U \in H^{\gamma}\left(S^{2 n+1}\right)$ is a critical point of $E$ if and only if $U$ is a weak solution of (FYS $)_{\gamma}$.

Let us fix $i \in\left\{1, \ldots,\left[\frac{n+1}{2}\right]\right\}$. In order to guarantee critical points for $E$, we first consider the functional $E_{i}: H_{\hat{G}_{i}}^{\gamma}\left(S^{2 n+1}\right) \rightarrow \mathbb{R}$, the restriction of $E$ to the space $H_{\hat{G}_{i}}^{\gamma}\left(S^{2 n+1}\right)$. It is clear that $E_{i}$ is an even functional and it has the mountain pass geometry. Since the embedding $H_{\hat{G}_{i}}^{\gamma}\left(S^{2 n+1}\right) \hookrightarrow L^{\frac{2 Q}{Q-2 \gamma}}\left(S^{2 n+1}\right)$ is compact, see Proposition 3.3 (i), we may apply the fountain theorem, see e.g. Bartsch and Willem [4, Theorem 3.1], guaranteeing a sequence $\left\{U_{i}^{k}\right\}_{k \in \mathbb{N}} \subset H_{\hat{G}_{i}}^{\gamma}\left(S^{2 n+1}\right)$ of critical points for $E_{i}$ with the additional property that $\left\|U_{i}^{k}\right\|_{H^{\gamma}} \rightarrow \infty$ as $k \rightarrow \infty$.

By using the principle of symmetric criticality of Palais [22], we are going to prove that $\left\{U_{i}^{k}\right\}_{k \in \mathbb{N}} \subset H_{\hat{G}_{i}}^{\gamma}\left(S^{2 n+1}\right)$ are in fact critical points for the original energy functional $E$, thus weak solutions of $(\mathbf{F Y S})_{\gamma}$. To do this, it suffices to verify that $E$ is a $\hat{G}_{i}$-invariant functional, i.e.,

$$
E(\hat{g} \circledast U)=E(U) \text { for every } \hat{g} \in \hat{G}_{i}, U \in H^{\gamma}\left(S^{2 n+1}\right) .
$$

On one hand, according to relation (2.6), for the quadratic term in $E$ it is enough to prove that $\hat{G}_{i}$ acts isometrically on $H^{\gamma}\left(S^{2 n+1}\right)$, i.e.,

$$
(\hat{g} \circledast U, \hat{g} \circledast U)_{\gamma}=(U, U)_{\gamma} \text { for every } \hat{g} \in \hat{G}_{i}, U \in H^{\gamma}\left(S^{2 n+1}\right) .
$$

To see this, let us fix $\hat{g} \in \hat{G}_{i}$ and $U \in H^{\gamma}\left(S^{2 n+1}\right)$ arbitrarily. We recall that by definition

$$
(\hat{g} \circledast U, \hat{g} \circledast U)_{\gamma}=\sum_{j, k \geq 0} \lambda_{j}(\gamma) \lambda_{k}(\gamma) \sum_{l=1}^{m_{j, k}}\left|c_{j, k}^{l}(\hat{g} \circledast U)\right|^{2} .
$$

By using (2.3), one has

$$
\begin{aligned}
\sum_{l=1}^{m_{j, k}}\left|c_{j, k}^{l}(\hat{g} \circledast U)\right|^{2} & =\int_{S^{2 n+1}} \int_{S^{2 n+1}}(\hat{g} \circledast U)(\zeta)(\hat{g} \circledast U)(\eta) \sum_{l=1}^{m_{j, k}} Y_{j, k}^{l}(\zeta) \overline{Y_{j, k}^{l}(\eta)} \mathrm{d} \zeta \mathrm{d} \eta \\
& =\int_{S^{2 n+1}} \int_{S^{2 n+1}}(\hat{g} \circledast U)(\zeta)(\hat{g} \circledast U)(\eta) \Phi_{j, k}(\zeta, \eta) \mathrm{d} \zeta \mathrm{d} \eta .
\end{aligned}
$$

Note that for every $g \in G_{i} \subset \mathbf{U}(n+1)$ and $\zeta, \eta \in S^{2 n+1}$ we have

$$
\langle g \zeta, \overline{g \eta}\rangle=\left\langle A_{i} g \zeta, \overline{A_{i} g \eta}\right\rangle=\langle\zeta, \bar{\eta}\rangle ;
$$

therefore, by the representation (2.4) of the zonal harmonics we also have that

$$
\Phi_{j, k}(g \zeta, g \eta)=\Phi_{j, k}\left(A_{i} g \zeta, A_{i} g \eta\right)=\Phi_{j, k}(\zeta, \eta) .
$$

Thus, relation (3.12) and suitable changes of variables in (3.14) imply that

$$
\sum_{l=1}^{m_{j, k}}\left|c_{j, k}^{l}(\hat{g} \circledast U)\right|^{2}=\int_{S^{2 n+1}} \int_{S^{2 n+1}} U(\zeta) U(\eta) \Phi_{j, k}(\zeta, \eta) \mathrm{d} \zeta \mathrm{d} \eta=\sum_{l=1}^{m_{j, k}}\left|c_{j, k}^{l}(U)\right|^{2},
$$

which proves (3.13).

On the other hand, the $\hat{G}_{i}$-invariance of the nonlinear term $U \mapsto \int_{S^{2 n+1}}|U|^{\frac{2 Q}{Q-2 \gamma}}$ trivially follows by a change of variable, by using the isometric character of the group $\mathbf{U}(n+1)$ on $S^{2 n+1}$.

Accordingly, for every $i \in\left\{1, \ldots,\left[\frac{n+1}{2}\right]\right\}$, the functions $\left\{U_{i}^{k}\right\}_{k \in \mathbb{N}} \subset H_{\hat{G}_{i}}^{\gamma}\left(S^{2 n+1}\right)$ are nontrivial weak solutions of $(\mathbf{F Y S})_{\gamma}$. Due to Proposition 3.1, $u_{i}^{k}=\left(2 \mathrm{Jac}_{\mathcal{C}}\right)^{\frac{Q-2 \gamma}{2 Q}} U_{i}^{k} \circ \mathcal{C} \in$ $D^{\gamma}\left(\mathbb{H}^{n}\right)$ are non-trivial weak solutions of the original fractional Yamabe problem $(\mathbf{F Y H})_{\gamma}$; by construction, $u_{i}^{k}$ are sign-changing functions. 
Due to Proposition 3.3 (iii), we state that the sequences $\left\{U_{i}^{k}\right\}_{k \in \mathbb{N}} \subset H_{\hat{G}_{i}}^{\gamma}\left(S^{2 n+1}\right)$ and $\left\{U_{j}^{k}\right\}_{k \in \mathbb{N}} \subset H_{\hat{G}_{j}}^{\gamma}\left(S^{2 n+1}\right)$ with $i, j \in\left\{1, \ldots,\left[\frac{n+1}{2}\right]\right\}, i \neq j$, cannot be compared from symmetrical point of view. Therefore, the sequences $\left\{u_{i}^{k}\right\} \subset D^{\gamma}\left(\mathbb{H}^{n}\right)$ and $\left\{u_{j}^{k}\right\} \subset D^{\gamma}\left(\mathbb{H}^{n}\right)$ have mutually different nodal properties for every $i, j \in\left\{1, \ldots,\left[\frac{n+1}{2}\right]\right\}, i \neq j$, which concludes the proof.

Remark 3.3. Consider a nonzero solution $u_{i}^{k}=\left(2 \operatorname{Jac}_{\mathcal{C}}\right)^{\frac{Q-2 \gamma}{2 Q}} U_{i}^{k} \circ \mathcal{C} \in D^{\gamma}\left(\mathbb{H}^{n}\right)$ of $(\mathbf{F Y H})_{\gamma}$, with $\left\{U_{i}^{k}\right\}_{k \in \mathbb{N}} \subset H_{\hat{G}_{i}}^{\gamma}\left(S^{2 n+1}\right) \backslash\{0\}$. For simplicity, we consider the case $n+1=2 i$. Let us introduce the nodal domain of $U_{i}^{k}$ (or $u_{i}^{k}$ ) as the connected components of $C_{i}^{k}=$ $S^{2 n+1} \backslash N_{i}^{k}$, where $N_{i}^{k}=\overline{\left\{\eta \in S^{2 n+1}: U_{i}^{k}(\eta)=0\right\}}$. Since $U_{i}^{k} \in H_{\hat{G}_{i}}^{\gamma}\left(S^{2 n+1}\right)$, by relation (3.12) it follows that $U_{i}^{k}$ has the form $U_{i}^{k}(\eta)=U_{i}^{k}\left(\left|\eta_{1}\right|,\left|\eta_{2}\right|\right)$ with the property that $U_{i}^{k}\left(\left|\eta_{1}\right|,\left|\eta_{2}\right|\right)=-U_{i}^{k}\left(\left|\eta_{2}\right|,\left|\eta_{1}\right|\right), \eta=\left(\eta_{1}, \eta_{2}\right) \in S^{2 n+1}, \eta_{1}, \eta_{2} \in \mathbb{C}^{i}$. Accordingly, since $U_{i}^{k}\left(\left|\eta_{1}\right|,\left|\eta_{2}\right|\right)=U_{i}^{k}\left(\left| \pm \eta_{1}\right|,\left| \pm \eta_{2}\right|\right), U_{i}^{k}$ is sign-changing with at least four non-degenerate nodal domains in $C_{i}^{k}$; in two of them the function $U_{i}^{k}$ is negative, while in the other two it is positive, respectively. When $n+1 \neq 2 i$, a similar discussion can be performed.

We conclude the paper by the following table providing explicit forms of subgroups of the unitary group $\mathbf{U}(n+1)$ and admissible intervals for the parameter $\gamma$, depending on the dimension $n$, where our main theorem applies; we only consider the cases when $n \in\{1, \ldots, 8\}$ :

\begin{tabular}{|c|c|c|c|c|}
\hline$n$ & $Q=2 n+2$ & $G_{i}, i \in\left\{1, \ldots,\left[\frac{n+1}{2}\right]\right\}$ & $\begin{array}{c}\text { Admissible domains } \\
\text { for } \gamma \in(0, Q / 2)\end{array}$ & $\begin{array}{c}\text { Number of symmetrically distinct } \\
\text { sequences of solutions of }(\mathbf{F Y H})_{\gamma}\end{array}$ \\
\hline 1 & 4 & $G_{1}=\mathbf{U}(1) \times \mathbf{U}(1)$ & {$\left[1, \frac{4}{3}\right)$} & 1 \\
\hline 2 & 6 & $G_{1}=\mathbf{U}(1) \times \mathbf{U}(1) \times \mathbf{U}(1)$ & {$\left[1, \frac{6}{5}\right) \cup\left[2, \frac{12}{5}\right)$} & 2 \\
\hline 3 & 8 & $\begin{array}{r}G_{1}=\mathbf{U}(1) \times \mathbf{U}(2) \times \mathbf{U}(1) \\
G_{2}=\mathbf{U}(2) \times \mathbf{U}(2)\end{array}$ & $\begin{array}{c}{\left[1, \frac{8}{7}\right) \cup\left[2, \frac{10}{7}\right) \cup} \\
\cup\left[3, \frac{24}{7}\right)\end{array}$ & \\
\hline 4 & 10 & $\begin{array}{l}G_{1}=\mathbf{U}(1) \times \mathbf{U}(3) \times \mathbf{U}(1) \\
G_{2}=\mathbf{U}(2) \times \mathbf{U}(1) \times \mathbf{U}(2)\end{array}$ & $\bigcup_{k=1}^{4}\left[k, \frac{10 k}{9}\right)$ & \\
\hline 5 & 12 & $\begin{array}{l}G_{1}=\mathbf{U}(1) \times \mathbf{U}(4) \times \mathbf{U}(1) \\
G_{2}=\mathbf{U}(2) \times \mathbf{U}(2) \times \mathbf{U}(2) \\
G_{3}=\mathbf{U}(3) \times \mathbf{U}(3)\end{array}$ & $\bigcup_{k=1}^{5}\left[k, \frac{12 k}{11}\right)$ & \\
\hline 6 & 14 & $\begin{array}{l}G_{1}=\mathbf{U}(1) \times \mathbf{U}(5) \times \mathbf{U}(1) \\
G_{2}=\mathbf{U}(2) \times \mathbf{U}(3) \times \mathbf{U}(2) \\
G_{3}=\mathbf{U}(3) \times \mathbf{U}(1) \times \mathbf{U}(3)\end{array}$ & $\bigcup_{k=1}^{6}\left[k, \frac{14 k}{13}\right)$ & \\
\hline 7 & 16 & $\begin{array}{l}G_{1}=\mathbf{U}(1) \times \mathbf{U}(6) \times \mathbf{U}(1) \\
G_{2}=\mathbf{U}(2) \times \mathbf{U}(4) \times \mathbf{U}(2) \\
G_{3}=\mathbf{U}(3) \times \mathbf{U}(2) \times \mathbf{U}(3) \\
G_{4}=\mathbf{U}(4) \times \mathbf{U}(4)\end{array}$ & $\bigcup_{k=1}^{7}\left[k, \frac{16 k}{15}\right)$ & \\
\hline 8 & 18 & $\begin{array}{l}G_{1}=\mathbf{U}(1) \times \mathbf{U}(7) \times \mathbf{U}(1) \\
G_{2}=\mathbf{U}(2) \times \mathbf{U}(5) \times \mathbf{U}(2) \\
G_{3}=\mathbf{U}(3) \times \mathbf{U}(3) \times \mathbf{U}(3) \\
G_{4}=\mathbf{U}(4) \times \mathbf{U}(1) \times \mathbf{U}(4)\end{array}$ & $\bigcup_{k=1}^{8}\left[k, \frac{18 k}{17}\right)$ & \\
\hline & & \\
\hline
\end{tabular}

Acknowledgment. The author would like to thank Professor Carlo Morpurgo for his useful remarks concerning the preliminary version of the manuscript and the anonymous referee for her/his valuable observations which improved the quality of the manuscript.

\section{REFERENCES}

[1] Th. Aubin, Nonlinear Analysis on Manifolds, Monge-Ampère equations, Springer-Verlag, New York, 1982. 
[2] Z. M. Balogh, A. Kristály, Lions-type compactness and Rubik actions on the Heisenberg group, Calc. Var. Partial Differential Equations 48 (2013), no. 1-2, 89-109.

[3] T. Bartsch, M. Schneider, T. Weth, Multiple solutions of a critical polyharmonic equation, J. Reine Angew. Math. 571 (2004), 131-143.

[4] T. Bartsch, M. Willem, Infinitely many nonradial solutions of a Euclidean scalar field equation, $J$. Funct. Anal. 117 (1993), no. 2, 447-460.

[5] T. P. Branson, L. Fontana, C. Morpurgo, Moser-Trudinger and Beckner-Onofri's inequalities on the CR sphere, Ann. of Math. 177 (2013), 1-52.

[6] X. Cabré, Y. Sire, Nonlinear equations for fractional Laplacians I: Regularity, maximum principles, and Hamiltonian estimates, Ann. Inst. H. Poincaré Anal. Non Linéaire 31 (2014), 23-53.

[7] L. Caffarelli, Nonlocal equations, drifts and games, Nonlinear Partial Differential Equations, Abel Symposia, 7 (2012), 37-52.

[8] L. Caffarelli, S. Salsa, L. Silvestre, Regularity estimates for the solution and the free boundary of the obstacle problem for the fractional Laplacian, Invent. Math. 171 (2008), 425-461.

[9] L. Caffarelli, L. Silvestre, An extension problem related to the fractional Laplacian, Comm. Partial Differential Equations 32 (2007), 1245-1260.

[10] E. Di Nezza, G. Palatucci, E. Valdinoci, Hitchhiker's guide to the fractional Sobolev spaces, Bull. Sci. Math. 136 (2012), 521-573.

[11] W. Y. Ding, On a conformally invariant elliptic equation, Comm. Math. Phys. 107 (1986), 331-335.

[12] G. B. Folland, A fundamental solution for a subelliptic operator, Bull. Amer. Math. Soc. 79 (1973), $373-376$.

[13] G. B. Folland, Spherical harmonics expansion of the Poisson-Szegö kernel for the ball, Proc. Amer. Math. Soc. 47 (1975), 401-408.

[14] R. L. Frank, E. H. Lieb, Sharp constants in several inequalities on the Heisenberg group, Ann. of Math. 176 (2012), 349-381.

[15] R. Frank, M. del Mar González, D. D. Monticelli, J. Tan, An extension problem for the CR fractional Laplacian, Adv. Math. 270 (2015), 97-137.

[16] N. Garofalo, D. Vassilev, Symmetry properties of positive entire solutions of Yamabe-type equations on groups of Heisenberg type, Duke Math. J. 106 (2001), no. 3, 411-448.

[17] E. Hebey, M. Vaugon, Sobolev spaces in the presence of symmetries, J. Math. Pures Appl. 76 (1997), 859-881.

[18] D. Jerison, J. M. Lee, The Yamabe problem on CR manifolds, J. Differential Geom. 25 (1987), 167-197.

[19] D. Jerison, J. M. Lee, Extremals for the Sobolev inequality on the Heisenberg group and the CR Yamabe problem, J. Amer. Math. Soc. 1 (1988), 1-13.

[20] A. Maalaoui, V. Martino, Changing-sign solutions for the CR-Yamabe equation, Differential Integral Equations 25 (2012), no. 7-8, 601-609.

[21] A. Maalaoui, V. Martino, G. Tralli, Complex group actions on the sphere and sign changing solutions for the CR-Yamabe equation, J. Math. Anal. Appl. 431 (2015), no. 1, 126-135.

[22] R. S. Palais, The principle of symmetric criticality, Comm. Math. Phys. 69 (1979), 19-30.

[23] N. Ja. Vilenkin, A. U. Klimyk, Representation of Lie groups and special functions. Vol. 2. Class I Representations, Special Functions, and Integral Transforms. Translated from the Russian by V. A. Groza and A. A. Groza. Mathematics and its Applications (Soviet Series), 74. Kluwer Academic Publishers Group, Dordrecht, 1993.

Department of Economics, Babeş-Bolyai University, 400591 Cluj-Napoca, Romania \& Institute of Applied Mathematics, ÓBuda University, 1034 Budapest, Hungary E-mail address: alexandrukristaly@yahoo.com; kristaly.alexandru@nik.uni-obuda.hu 